\title{
First-principles investigation of transient dynamics of molecular devices
}

\author{
Lei Zhang, ${ }^{1}$ Yanxia Xing, ${ }^{1,2}$ and Jian Wang ${ }^{1, *}$ \\ ${ }^{1}$ Department of Physics and the Center of Theoretical and Computational Physics, The University of Hong Kong, Hong Kong, China \\ ${ }^{2}$ Department of Physics, Beijing Institute of Technology, Beijing 100081, China
}

(Received 29 June 2012; published 19 October 2012)

\begin{abstract}
Based on the nonequilibrium Green's function (NEGF) and time-dependent density-functional theory (TDDFT), we propose a formalism to study the time-dependent transport behavior of molecular devices from first principles. While this approach is equivalent to the time-dependent wave-function approach within TDDFT, it has the advantage that the scattering states and bound states are treated on equal footing. Furthermore, it is much easier to implement our approach numerically. Different from the time-dependent wave-function $[\psi(t, E)]$ approach, our formalism is in the time space $\left[\mathbf{G}^{r}\left(t, t^{\prime}\right)\right.$ ], making this method superior in the time-dependent transport problem with many subbands in the transverse direction. For the purpose of numerical implementation on molecular devices, a computational tractable numerical scheme is discussed in detail. We have applied our formalism to calculate the transient current of two molecular devices Al-1,4-dimethylbenzene-Al and Al-benzene-Al from first principles. In the calculation, we have gone beyond the wideband limit and used the adiabatic local density approximation that was used within TDDFT. It is known that when the wideband limit is abandoned, the boundary condition of the transport problem is non-Markovian, resulting in a memory term in the effective Hamiltonian of the scattering region. To overcome the computational complexity due to the memory term, we have employed a fast algorithm to speed up the calculation and reduced the CPU time from the scaling $N^{3}$ to $N^{2} \log _{2}^{2}(N)$ for the steplike pulse, where $N$ is the number of time steps in the time evolution of the Green's function. To ensure the accuracy of our method, we have done a benchmark transient calculation on an atomic junction using a time-dependent wave-function approach within TDDFT in momentum space, which agrees very well with the result from our method.
\end{abstract}

DOI: 10.1103/PhysRevB.86.155438

PACS number(s): 73.63.-b, 72.30.+q, 71.15.Mb

\section{INTRODUCTION}

Advanced by the recent experimental progress in the fabrication and characterization of nanostructures, quantum transport in coherent nanoelectronic devices both in experimental and theoretical sides attracts a lot of research efforts. ${ }^{1-10}$ Because these ultrasmall nanoelectronic devices are essentially different from the traditional microelectronic devices due to the quantum effect, people have to carry out density-functional theory (DFT) calculations combined within the nonequilibrium Green's functions (NEGF) from first principles to predict their transport properties. ${ }^{9-13}$ Currently, NEGF-DFT formalism is widely used in calculating physical quantities when the system reaches the steady-state regime and quantitative comparisons to experimental data have been presented. ${ }^{14-18}$ However, the transient dynamical response of nanoelectronic devices is still a serious challenge. Strictly speaking, one has to combine quantum transport theory such as the scattering approach or NEGF approach with timedependent density-functional theory (TDDFT) to predict the dynamical transport properties. ${ }^{19-22}$ However, it is extremely difficult to implement it exactly from first principles from the numerical point of view. In practice, people usually take the wideband limit (WBL) to accelerate numerical calculation. ${ }^{23}$ For a pulselike bias voltage, an exact solution exists for transient current from NEGF theory ${ }^{24}$ which in principle can be coupled to DFT or TDDFT for first-principles calculation. Due to the computational complexity of the exact solution, a practical scheme has been proposed and applied to calculate the transient current of atomic junctions. ${ }^{25}$ While many firstprinciples calculations for the transient current of nanodevices have been done with various approximations used, it will be highly desirable to carry out a numerical exact first-principles calculation for the transient current of molecular devices. It is the purpose of this paper to provide such a kind of numerical exact first-principles calculation.

Before proceeding further, we wish to discuss some basic issues concerning the quantum transport formalism of nanodevices. For an open system, the Hamiltonian can be divided into three different parts: (1) the isolated central region (molecular device region) and external leads, (2) the coupling between the central region and leads, (3) the time-dependent external bias applied on the leads. Historically, there exist two different approaches depending on the choice of the unperturbed Hamiltonian of the system. One is called the "partitioned approach," which was first proposed by Caroli et al. ${ }^{26-29} \mathrm{In}$ this approach, the central and lead regions are separated in the remote past, and each region is in its thermodynamic equilibrium, respectively. In this approach, one assumes that the time-dependent external bias is already applied on the lead in the remote past. The perturbation, the coupling between the central region and leads, is adiabatically switched on. This approach has been widely used to predict transport properties for mesoscopic systems. ${ }^{30}$ In addition, many first-principles calculations based on NEGF-DFT formalism are also in the framework of the "partitioned approach."9-13,23

Several years after the birth of the partitioned approach, Cini proposed another scheme ${ }^{31}$ to study the quantum transport problem, which is called the "partition-free approach." $\mathrm{He}$ assumed that the central region is connected to the external leads and they are in the thermodynamic equilibrium in the remote past. The time-dependent perturbation such as time-dependent external bias is turned on in the leads at a 
particular time $t=t_{0}$. Different from the partitioned approach, the time-independent coupling Hamiltonian is treated as the unperturbed one. It was argued by Cini that the transient response due to the time-dependent external bias is more realistic in this approach compared with the real experimental setup. In other words, the partition-free approach is well suited to study the transient response or time-dependent behavior of molecular devices due to the external bias perturbation. Most of the studies on transient dynamics used the partition-free approach. . $^{21,24,25,32,33}$

In this paper, we propose a general NEGF-TDDFT formalism to calculate transient current based on the partition-free approach. Our formalism is equivalent to the time-dependent wave-function approach within TDDFT proposed by Kurth et $a l .{ }^{21}$ One of the advantages of our approach is that the scattering states and the bound states are treated on equal footing so that it is very convenient to determine the nonequilibrium Coulomb interaction self-consistently. Importantly, our method is in the time domain which is particularly useful for the problem with many subbands in the transverse direction. We have implemented this approach in the first-principles calculation for transient transport in molecular devices. In particular, we have calculated the transient current for the steplike bias in molecular devices such as Al-1,4 dimethylbenzene (para-xylene)-Al and Al-benzene-Al without making wideband-limit approximation or other kinds of approximations in the transport part of the calculation. ${ }^{34} \mathrm{~A}$ fast algorithm has been employed to speed up the calculation so that the CPU time needed for transient current versus the number of time steps $N$ is reduced from the scaling $N^{3}$ to $N^{2} \log _{2}^{2}(N)$, a huge gain in speed for large $N .^{35}$ To test the accuracy of this method, we have also calculated the transient current by solving the Schrödinger equation coupled with TDDFT in the momentum space for a one-dimensional carbon chain. Excellent agreement is obtained between results obtained by the two methods.

The paper is organized as follows. In Sec. II, we describe the NEGF-TDDFT formalism for studying the time-dependent quantum transport of molecular devices. We have also shown that it is equivalent to the time-dependent wave-function approach within TDDFT. In Sec. III, computational details to transient current of open molecular devices are given. $\mathrm{Nu}$ merical results of molecular devices Al-1,4 dimethylbenzene (para-xylene)-Al and Al-benzene-Al under a upward pulse are presented in Sec. IV. We have also discussed the algorithm to speed up the calculation. Section V serves as a discussion and conclusion part.

\section{THEORETICAL FORMALISM}

We begin with a typical two-terminal device shown in Fig. 1, which consists of the central scattering region connected by two external leads. We assume that $\mathbf{H}(t)$ is the time-dependent Kohn-Sham (KS) Hamiltonian of the entire two-terminal device. In the framework of the partition-free approach, the time-dependent bias $\mathbf{V}_{\alpha}(t)$ is turned on in both leads at $t=0$ when the open device is in equilibrium, i.e., $\mathbf{H}_{\alpha \alpha}(t)=$ $\mathbf{H}_{\alpha \alpha}(0)-\mathbf{V}_{\alpha}(t), \alpha=L, R$ [atomic units (a.u.) $e=\hbar=1$ are used]. Therefore, the equation of motion for the retarded Green's function is given by

$$
\left[\begin{array}{ccc}
i \frac{\partial}{\partial t}-\mathbf{H}_{L L}(t) & -\mathbf{H}_{L C} & 0 \\
-\mathbf{H}_{C L} & i \frac{\partial}{\partial t}-\mathbf{H}_{C C}(t) & -\mathbf{H}_{C R} \\
0 & -\mathbf{H}_{R C} & i \frac{\partial}{\partial t}-\mathbf{H}_{R R}(t)
\end{array}\right]\left[\begin{array}{ccc}
\mathbf{G}_{L L}^{r}\left(t, t^{\prime}\right) & \mathbf{G}_{L C}^{r}\left(t, t^{\prime}\right) & \mathbf{G}_{L R}^{r}\left(t, t^{\prime}\right) \\
\mathbf{G}_{C L}^{r}\left(t, t^{\prime}\right) & \mathbf{G}_{C C}^{r}\left(t, t^{\prime}\right) & \mathbf{G}_{C R}^{r}\left(t, t^{\prime}\right) \\
\mathbf{G}_{R L}^{r}\left(t, t^{\prime}\right) & \mathbf{G}_{R C}^{r}\left(t, t^{\prime}\right) & \mathbf{G}_{R R}^{r}\left(t, t^{\prime}\right)
\end{array}\right]=\delta\left(t-t^{\prime}\right) \mathbf{I},
$$

where $\mathbf{G}_{\alpha \beta}^{r}\left(t, t^{\prime}\right)$ is the retarded Green's function projected on three different regions $\alpha, \beta=L, C, R$ and $\mathbf{I}$ is the unit matrix. Since the time-dependent bias $\mathbf{V}_{\alpha}(t)$ is applied on the lead, the central scattering region's Hamiltonian $\mathbf{H}_{C C}(t)$ also depends on time and the couplings between Hamiltonian in the central region and leads are assumed to be time independent. To make this open boundary problem tractable, we focus on the central scattering region, which provides us enough information for transport properties. From Eq. (1), an effective equation only involving the central scattering region is obtained:

$\left[i \frac{\partial}{\partial t}-\mathbf{H}_{C C}(t)\right] \mathbf{G}_{C C}^{r}\left(t, t^{\prime}\right)-\sum_{\alpha=L, R} \mathbf{H}_{C \alpha} \mathbf{G}_{\alpha C}^{r}\left(t, t^{\prime}\right)=\delta\left(t-t^{\prime}\right)$

and

$$
\left[i \frac{\partial}{\partial t}-\mathbf{H}_{\alpha \alpha}(t)\right] \mathbf{G}_{\alpha C}^{r}\left(t, t^{\prime}\right)-\mathbf{H}_{\alpha C} \mathbf{G}_{C C}^{r}\left(t, t^{\prime}\right)=0 .
$$

Note that Eq. (3) is an inhomogeneous differential equation, the solution $\mathbf{g}_{\alpha \alpha}^{r}\left(t, t^{\prime}\right)$ of which can be expressed in terms of the general solution of corresponding homogeneous equation $\tilde{\mathbf{G}}_{\alpha C}^{r}\left(t, t^{\prime}\right)$ as follows:

$$
\mathbf{G}_{\alpha C}^{r}\left(t, t^{\prime}\right)=\tilde{\mathbf{G}}_{\alpha C}^{r}\left(t, t^{\prime}\right)+\int_{0}^{t} \mathbf{g}_{\alpha \alpha}^{r}\left(t, t_{1}\right) \mathbf{H}_{\alpha C} \mathbf{G}_{C C}^{r}\left(t_{1}, t^{\prime}\right) d t_{1},
$$

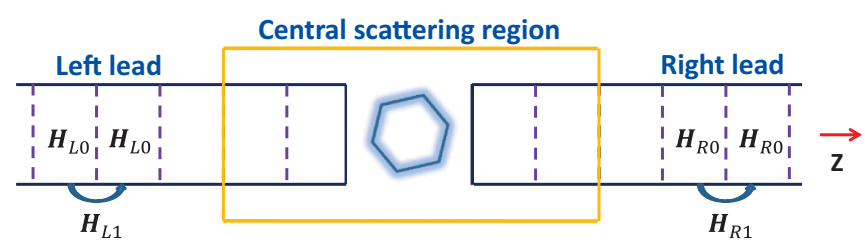

FIG. 1. (Color online) A sketch illustrating the typical twoterminal molecular device which is sandwiched between two semiinfinite external leads. The central scattering region contains a molecular device and several buffer layers of leads. Left and right leads are assumed to be periodic in the transport $z$ direction. $\mathbf{H}_{\alpha 0}$ is the unit-cell matrix and $\mathbf{H}_{\alpha 1}$ describes the interaction between the nearest unit cell. 
where the retarded Green's function $\mathbf{g}_{\alpha \alpha}^{r}\left(t, t^{\prime}\right)$ of the $\alpha$ lead is defined as

$$
\left[i \frac{\partial}{\partial t}-\mathbf{H}_{\alpha \alpha}(t)\right] \mathbf{g}_{\alpha \alpha}^{r}\left(t, t^{\prime}\right)=\delta\left(t-t^{\prime}\right)
$$

subjected to the boundary condition $\mathbf{g}_{\alpha \alpha}^{r}\left(t^{+}, t\right)=-i$ and $\mathbf{g}_{\alpha \alpha}^{r}\left(t, t^{+}\right)=0$. The Green's function $\tilde{\mathbf{G}}_{\alpha C}^{r}\left(t, t^{\prime}\right)$ in Eq. (4) can be written as

$\tilde{\mathbf{G}}_{\alpha C}^{r}\left(t, t^{\prime}\right)=i \mathbf{g}_{\alpha \alpha}^{r}(t, 0) \tilde{\mathbf{G}}_{\alpha C}^{r}\left(0, t^{\prime}\right)=i \mathbf{g}_{\alpha \alpha}^{r}(t, 0) \mathbf{G}_{\alpha C}^{r}\left(0, t^{\prime}\right)$.

Since $\mathbf{G}_{\alpha C}^{r}\left(0, t^{\prime}\right)=0$ for $t^{\prime}>0$ and $\mathbf{G}_{\alpha C}^{r}\left(0^{+}, 0\right)=0$, from Eq. (6) we see that the first term in Eq. (4) is zero. Finally, plugging Eq. (4) into Eq. (2), we have

$$
\begin{aligned}
& {\left[i \frac{\partial}{\partial t}-\mathbf{H}_{C C}(t)\right] \mathbf{G}_{C C}^{r}\left(t, t^{\prime}\right)} \\
& \quad=\delta\left(t-t^{\prime}\right)+\int_{0}^{t} \boldsymbol{\Sigma}^{r}\left(t, t_{1}\right) \mathbf{G}_{C C}^{r}\left(t_{1}, t^{\prime}\right) d t_{1},
\end{aligned}
$$

where the self-energy $\boldsymbol{\Sigma}^{r}\left(t, t^{\prime}\right) \equiv \sum_{\alpha=L, R} \mathbf{H}_{C \alpha} \mathbf{g}_{\alpha \alpha}^{r}\left(t, t^{\prime}\right) \mathbf{H}_{\alpha C}$ is introduced. Note that the self-energy $\boldsymbol{\Sigma}^{r}\left(t, t^{\prime}\right)$ is a function of double-time indices in the presence of time-dependent bias. Equation (7) is obtained in the partition-free approach. The same equation has been obtained in the partitioned approach, ${ }^{36}$ the only difference is the lower limit of integration (from $-\infty$ ) in the second term.

Now, we find the equation for the time-dependent charge density in the scattering region since it determines both the self-consistent induced Coulomb potential and the transient current. To do that, one has to know the equation of motion of the lesser Green's function. It can be easily obtained by multiplying $\left[\boldsymbol{\Sigma}^{<} \mathbf{G}_{C C}^{a}\right]\left(t, t^{\prime}\right)$ from the right-hand side of Eq. (7):

$$
\begin{aligned}
i \frac{\partial}{\partial t} \mathbf{G}_{C C}^{<}\left(t, t^{\prime}\right)= & \mathbf{H}_{C C}(t) \mathbf{G}_{C C}^{<}\left(t, t^{\prime}\right)+\int_{0}^{t}\left[\boldsymbol{\Sigma}^{<}\left(t, t_{1}\right) \mathbf{G}_{C C}^{a}\left(t_{1}, t^{\prime}\right)\right. \\
& \left.+\boldsymbol{\Sigma}^{r}\left(t, t_{1}\right) \mathbf{G}^{<}\left(t_{1}, t^{\prime}\right)\right] d t_{1} .
\end{aligned}
$$

Practically, it is enough for us to know the dynamics of charge density through calculating lesser Green's function $\mathbf{G}_{C C}^{<}(t, t)$. On the other hand, we can calculate it using the Keldysh equation instead of solving Eq. (8):

$$
\begin{aligned}
\mathbf{G}_{C C}^{<}(t, t)= & \mathbf{G}_{C C}^{r}(t, 0) \mathbf{g}_{C C}^{<}(0,0) \mathbf{G}_{C C}^{a}(0, t) \\
& +\int_{0}^{t} d t_{1} d t_{2} \mathbf{G}_{C C}^{r}\left(t, t_{1}\right) \boldsymbol{\Sigma}^{<}\left(t_{1}, t_{2}\right) \mathbf{G}_{C C}^{a}\left(t_{2}, t\right),
\end{aligned}
$$

where $\mathbf{g}_{C C}^{<}(0,0)$ is the initial lesser Green's function for the discrete bound states of the system. Bound states are essential to obtain correct time-dependent charge density $\rho$, which in turn affects the time-dependent potential landscape if a self-consistent Hartree potential is included. However, they do not contribute directly to the time-dependent terminal current $I_{\alpha}(t)$. This is because in a real experiment the terminal current is measured in two external leads which are far away from the central region, while bound states are localized in the central scattering region. From the computational point of view, the first term in Eq. (9) is much easier to handle and can be treated separately from the time evolution of the retarded Green's function. To calculate the second term in Eq. (9) at $t>t^{\prime}$, it is more efficient to iterate the retarded Green's function, Eq. (7).
In view of Eq. (9), it is enough to calculate part of the retarded Green's function defined as $\overline{\mathbf{G}}_{C C \alpha}^{r}\left(t, t^{\prime}\right)=\mathbf{G}_{C C}^{r}\left(t, t^{\prime}\right) \bar{\Gamma}_{\alpha}$, where we also have introduced two auxiliary projection matrices

$$
\overline{\boldsymbol{\Gamma}}_{L}=\left[\begin{array}{ccc}
\mathbf{I}_{L} & 0 & 0 \\
0 & 0 & 0 \\
0 & 0 & 0
\end{array}\right], \quad \overline{\boldsymbol{\Gamma}}_{R}=\left[\begin{array}{ccc}
0 & 0 & 0 \\
0 & 0 & 0 \\
0 & 0 & \mathbf{I}_{R}
\end{array}\right],
$$

where $\mathbf{I}_{L / R}$ is the unit matrix with dimension equal to the size of the unit cell of the left and right leads, respectively.

From Eq. (7) we have the equation for $\overline{\mathbf{G}}_{C C \alpha}^{r}\left(t, t^{\prime}\right)$,

$$
\begin{aligned}
& i \frac{\partial}{\partial t} \overline{\mathbf{G}}_{C C \alpha}^{r}\left(t, t^{\prime}\right) \\
& \quad=\mathbf{H}_{C C}(t) \overline{\mathbf{G}}_{C C \alpha}^{r}\left(t, t^{\prime}\right)+\int_{0}^{t} \boldsymbol{\Sigma}^{r}\left(t, t_{1}\right) \overline{\mathbf{G}}_{C C \alpha}^{r}\left(t_{1}, t^{\prime}\right) d t_{1},
\end{aligned}
$$

which can be calculated iteratively for fixed time $t$ and $t^{\prime}$. To calculate the second term of $\mathbf{G}_{C C}^{<}(t, t)$ at time $t=N \Delta \tau$ ( $N$ is the number of time steps), one has to know the information of $\overline{\mathbf{G}}_{C C \alpha}^{r}(N \Delta \tau, n \Delta \tau)$ where $n$ varies from 0 to $N$. We fix the time $t^{\prime}=n \Delta \tau$ and iterate Eq. (11) where the time $t$ goes from $n \Delta \tau$ to $N \Delta \tau$. This enables us to find the series of $\overline{\mathbf{G}}_{C C \alpha}^{r}(N \Delta \tau, n \Delta \tau)$ where $n=0, \ldots, N$. After it is done, the time-dependent charge density at each time $t$ is obtained from Eq. (9) and the time-dependent Hartree potential $\mathbf{U}_{H}(t)$ can be calculated from

$$
\nabla^{2} \mathbf{U}_{H}(t)=-4 \pi \rho(\mathbf{r}, t)=4 \pi i \mathbf{G}_{C C}^{<}(t, t) .
$$

Once Eq. (12) is solved, the time-dependent Hamiltonian can be updated:

$$
\mathbf{H}_{C C}(t)=\mathbf{H}_{C C}^{0}+\mathbf{U}_{H}(t)+\mathbf{U}_{X C}(t),
$$

where $\mathbf{H}_{C C}^{0}$ is the time-independent Hamiltonian excluding exchange correlation and Hartree potential. Here, $\mathbf{U}_{X C}(t)$ is the time-dependent exchange-correlation potential and can be chosen as an adiabatic local density approximation (LDA) form. It has been confirmed numerically that TDDFT within the adiabatic LDA gives the same steady-state current as that from the Landauer-Büttiker formula. ${ }^{37}$ Finally, we can calculate the time-dependent current by using the time evolution of the lesser Green's function $\mathbf{G}_{C C}^{<}(t, t)$ (Ref. 33):

$$
I_{\alpha}(t)=2 \operatorname{Re} \operatorname{Tr}\left[\overline{\boldsymbol{\Gamma}}_{\alpha} \mathbf{H}_{C C}(t) \mathbf{G}_{C C}^{<}(t, t) \overline{\boldsymbol{\Gamma}}_{\alpha}\right] .
$$

To summarize, the above formalism is exact based on NEGF coupled with TDDFT formalism to calculate time-dependent current of the two-terminal device with the Poisson equation (12) solved self-consistently.

It is extremely time consuming to calculate the transient current using the above formalism. However, it is less computationally demanding if the self-consistent Coulomb interaction due to the time-dependent bias is not calculated. To avoid this self-consistent calculation, we assume that $\mathbf{U}_{H}(t)=\theta(t) \mathbf{U}_{1}(x)$ where $\mathbf{U}_{1}(x)$ is the self-consistent Coulomb potential of the system under the bias $V_{L}-V_{R}$, i.e., the potential landscape when $t$ goes to infinity. Under this approximation, we do not have to worry about the bound states in the system and hence the scattering states are enough to characterize the transport properties. Therefore, one may use the scattering wave function instead of the Green's function. In the following, we will derive an effective Schrödinger equation for the 
wave function in the central scattering region from Green's functions. According to the definition of Green's function $\left[\psi(t)=i \mathbf{G}^{r}\left(t, t^{\prime}\right) \psi\left(t^{\prime}\right)\right]$, the time-dependent wave function in the central scattering region $\psi_{C}(t)$ can be expressed as

$$
\psi_{C}(t)=i \mathbf{G}_{C C}^{r}(t, 0) \psi_{C}(0)+\sum_{\alpha=L, R} i \mathbf{G}_{C \alpha}^{r}(t, 0) \psi_{\alpha}(0),
$$

where $\psi_{\beta}(0), \beta=L, C, R$ is the initial wave function projected on each region of the whole system. From Eq. (1), we have the equation for $\mathbf{G}_{C \alpha}^{r}(t, 0)$ :

$$
\begin{aligned}
& {\left[i \frac{\partial}{\partial t}-\mathbf{H}_{C C}(t)\right] \mathbf{G}_{C \alpha}^{r}(t, 0)} \\
& \quad=\mathbf{H}_{C \alpha}(t) \mathbf{g}_{\alpha \alpha}^{r}(t, 0)+\int_{0}^{t} \boldsymbol{\Sigma}^{r}\left(t, t_{1}\right) \mathbf{G}_{C \alpha}^{r}\left(t_{1}, 0\right) .
\end{aligned}
$$

Combining Eqs. (7) and (16) with the help of Eq. (15), we arrive at

$$
\begin{aligned}
& {\left[i \frac{\partial}{\partial t}-\mathbf{H}_{C C}(t)\right] \psi_{C}(t)} \\
& \quad=i \sum_{\alpha=L, R} \mathbf{H}_{C \alpha}(t) \mathbf{g}_{\alpha \alpha}^{r}(t, 0) \psi_{\alpha}(0)+\int_{0}^{t} d t_{1} \boldsymbol{\Sigma}^{r}\left(t, t_{1}\right) \psi_{C}\left(t_{1}\right),
\end{aligned}
$$

which was first obtained in Ref. 38 and then discussed in detail in Ref. 21.

As mentioned above, only scattering states are needed to calculate the time-dependent current if the self-consistent Coulomb interaction is neglected. In terms of scattering wave function, the time-dependent terminal current $I_{\alpha}(t)$ can be written as 21

$$
I_{\alpha}(t)=-2 \sum_{E_{k^{n}}} f\left(E_{k^{n}}\right) \operatorname{Im} \operatorname{Tr}\left[\overline{\boldsymbol{\Gamma}}_{\alpha} \mathbf{H}_{C C}(t)\left|\psi_{C}^{k^{n}}(t)\right\rangle\left\langle\psi_{C}^{k^{n}}(t)\right| \overline{\boldsymbol{\Gamma}}_{\alpha}\right],
$$

where $\left|\psi_{C}^{k^{n}}\right\rangle$ is the scattering state of open device with $k$ and $n$ denoting the wave vector and band number of the incident wave function; $E_{k^{n}}$ is the corresponding eigenenergy of the state $\left|\psi_{C}^{k^{n}}\right\rangle$ and $f\left(E_{k^{n}}\right)$ is the Fermi distribution function.

\section{COMPUTATION TECHNIQUES}

In this section, we discuss how to numerically evolve the time-dependent retarded Green's function in Eq. (12) and hence calculate the time-dependent current in detail. Since the direct discretization of time in Eq. (12) can result in a norm-conserving problem, we start from the discrete form of Green's function by using the Crank-Nicholson scheme to obtain an iterative equation that satisfies the unitary condition ${ }^{21}$

$$
\begin{aligned}
& {\left[\begin{array}{ccc}
\mathbf{I}_{L}+i \tau \mathbf{H}_{L L}^{(0)} & i \tau \mathbf{H}_{L C} & 0 \\
i \tau \mathbf{H}_{C L} & \mathbf{I}_{C}+i \tau \mathbf{H}_{C C}^{(m)} & i \tau \mathbf{H}_{C R} \\
0 & i \tau \mathbf{H}_{R C} & \mathbf{I}_{R}+i \tau \mathbf{H}_{R R}^{(0)}
\end{array}\right]\left[\begin{array}{ccc}
\frac{\mathbf{I}_{L}-i \frac{\tau}{2} \mathbf{V}_{L}^{(m)}}{\mathbf{I}_{L}+i \frac{\tau}{2} \mathbf{V}_{L}^{(m)}} & 0 & 0 \\
0 & \mathbf{I}_{C} & 0 \\
0 & 0 & \frac{\mathbf{I}_{R}-i \frac{\tau}{2} \mathbf{V}_{m}^{(m)}}{\mathbf{I}_{R}+i \frac{\tau}{2} \mathbf{V}_{R}^{(m)}}
\end{array}\right]\left[\begin{array}{ccc}
\mathbf{G}_{L L}^{(m+1, n)} & \mathbf{G}_{L C}^{(m+1, n)} & \mathbf{G}_{L R}^{(m+1, n)} \\
\mathbf{G}_{C L}^{(m+1, n)} & \mathbf{G}_{C C}^{(m+1, n)} & \mathbf{G}_{C R}^{(m+1, n)} \\
\mathbf{G}_{R L}^{(m+1, n)} & \mathbf{G}_{R C}^{(m+1, n)} & \mathbf{G}_{R R}^{(m+1, n)}
\end{array}\right]} \\
& =\left[\begin{array}{ccc}
\mathbf{I}_{L}-i \tau \mathbf{H}_{L L}^{(0)} & -i \tau \mathbf{H}_{L C} & 0 \\
-i \tau \mathbf{H}_{C L} & \mathbf{I}_{C}-i \tau \mathbf{H}_{C C}^{(m)} & -i \tau \mathbf{H}_{C R} \\
0 & -i \tau \mathbf{H}_{R C} & \mathbf{I}_{R}-i \tau \mathbf{H}_{R R}^{(0)}
\end{array}\right]\left[\begin{array}{ccc}
\frac{\mathbf{I}_{L}+i \frac{\tau}{2} \mathbf{V}_{L}^{(m)}}{\mathbf{I}_{L}-i \frac{\tau}{2} \mathbf{V}_{L}^{(m)}} & 0 & 0 \\
0 & \mathbf{I}_{C} & 0 \\
0 & 0 & \frac{\mathbf{I}_{R}+i \frac{\tau}{2} \mathbf{V}_{R}^{(m)}}{\mathbf{I}_{R}-i \frac{\tau}{2} \mathbf{V}_{R}^{(m)}}
\end{array}\right]\left[\begin{array}{ccc}
\mathbf{G}_{L L}^{(m, n)} & \mathbf{G}_{L C}^{(m, n)} & \mathbf{G}_{L R}^{(m, n)} \\
\mathbf{G}_{C L}^{(m, n)} & \mathbf{G}_{C C}^{(m, n)} & \mathbf{G}_{C R}^{(m, n)} \\
\mathbf{G}_{R L}^{(m, n)} & \mathbf{G}_{R C}^{(m, n)} & \mathbf{G}_{R R}^{(m, n)}
\end{array}\right],
\end{aligned}
$$

where $\mathbf{G}_{\alpha \beta}^{(m, n)}$ denotes the retarded Green's function $\mathbf{G}_{\alpha \beta}^{r}(t=$ $m \Delta t, t^{\prime}=n \Delta t$ ) with $m>n$. For $m=n$, we have the initial condition $\mathbf{G}_{\alpha \beta}^{(n, n)}=-i \delta_{\alpha \beta}$. In the above equation, we adopt the following notation: $\mathbf{H}^{(m)}=\left[\mathbf{H}\left(t_{m+1}\right)+\mathbf{H}\left(t_{m}\right)\right] / 2$ and $\mathbf{V}_{\beta}^{(m)}=$ $\left[\mathbf{V}_{\beta}\left(t_{m+1}\right)+\mathbf{V}_{\beta}\left(t_{m}\right)\right] / 2 ; t_{m}=m \Delta t \tau=\Delta t / 2$. Therefore, we can obtain two discretized versions of Eqs. (2) and (16):

$$
\begin{gathered}
\left(\mathbf{I}_{C}+i \tau \mathbf{H}_{C C}^{(m)}\right) \mathbf{G}_{C \alpha}^{(m+1, n)}+i \tau \sum_{\beta=L, R} \mathbf{H}_{C \beta}\left(u_{\beta}^{(m)}\right)^{-1} \mathbf{G}_{\beta \alpha}^{(m+1, n)} \\
=\left(\mathbf{I}_{C}-i \tau \mathbf{H}_{C C}^{(m)}\right) \mathbf{G}_{C \alpha}^{(m, n)}-i \tau \sum_{\beta=L, R} \mathbf{H}_{C \beta} u_{\beta}^{(m)} \mathbf{G}_{\beta \alpha}^{(m, n)}
\end{gathered}
$$

and

$$
\begin{gathered}
\left(\mathbf{I}_{\beta}+i \tau \mathbf{H}_{\beta \beta}^{(0)}\right)\left(u_{\beta}^{(m)}\right)^{-1} \mathbf{G}_{\beta \alpha}^{(m+1, n)}+i \tau \mathbf{H}_{\beta C} \mathbf{G}_{C \alpha}^{(m+1, n)} \\
=\left(\mathbf{I}_{\beta}-i \tau \mathbf{H}_{\beta \beta}^{(0)}\right) u_{\beta}^{(m)} \mathbf{G}_{\beta \alpha}^{(m, n)}-i \tau \mathbf{H}_{\beta C} \mathbf{G}_{C \alpha}^{(m, n)}
\end{gathered}
$$

where we have introduced

$$
u_{\beta}^{(m)}=\frac{1+i \frac{\tau}{2} V_{\beta}^{(m)}}{1-i \frac{\tau}{2} V_{\beta}^{(m)}}
$$

where we have used the fact that $\mathbf{V}_{\beta}^{(m)}$ is a diagonal matrix. In Eqs. (20) and (21), $\alpha$ can be $L, C, R$ and $\beta$ can only take $L, R$. Plugging $\mathbf{G}_{\beta \alpha}^{(m+1, n)}$ from Eq. (21) into Eq. (20), we obtain an effective equation

$$
\begin{aligned}
\left(\mathbf{I}_{C}\right. & \left.+i \tau \mathbf{H}_{\mathrm{eff}}^{(m)}\right) \mathbf{G}_{C \alpha}^{(m+1, n)} \\
= & \left(\mathbf{I}_{C}-i \tau \mathbf{H}_{\mathrm{eff}}^{(m)}\right) \mathbf{G}_{C \alpha}^{(m, n)} \\
& -i \tau \sum_{\beta=L, R} \mathbf{H}_{C \beta} \frac{2}{\mathbf{I}_{\beta}+i \tau \mathbf{H}_{\beta \beta}^{(0)}} u_{\beta}^{(m)} \mathbf{G}_{\beta \alpha}^{(m, n)}
\end{aligned}
$$


with an effective Hamiltonian

$$
\mathbf{H}_{\mathrm{eff}}^{(m)}=\mathbf{H}_{C C}^{(m)}-i \tau \sum_{\beta=L, R} \mathbf{H}_{C \beta}\left(\mathbf{I}_{\beta}+i \tau \mathbf{H}_{\beta \beta}^{(0)}\right)^{-1} \mathbf{H}_{\beta C} .
$$

However, Eq. (23) is still not convenient to do iteration due to the unknown term $\mathbf{G}_{\beta \alpha}^{(m, n)}$. By using the recursive method, ${ }^{21}$ we can express $\mathbf{G}_{\beta \alpha}^{(m, n)}$ in terms of the initial Green's function $\mathbf{G}_{\beta \alpha}^{(n, n)}$ and $\mathbf{G}_{C \alpha}^{(m, n)}$. Finally, we arrive at an effective equation for $\mathbf{G}_{C \alpha}^{(m, n)}$ :

$$
\begin{aligned}
& \left(\mathbf{I}_{C}+i \tau \mathbf{H}_{\mathrm{eff}}^{(m)}\right) \mathbf{G}_{C \alpha}^{(m+1, n)} \\
& \quad=\left(\mathbf{I}_{C}-i \tau \mathbf{H}_{\mathrm{eff}}^{(m)}\right) \mathbf{G}_{C \alpha}^{(m, n)}+\mathbf{F}_{1 \alpha}^{(m, n)}+\mathbf{F}_{2 \alpha}^{(m, n)},
\end{aligned}
$$

where two auxiliary terms are defined as

$$
\mathbf{F}_{1 \alpha}^{(m, n)}=-2 i \tau \sum_{\beta=L, R} \frac{\Lambda_{\beta}^{(m, 0)}}{u_{\beta}^{(m)}} \mathbf{H}_{C \beta} \frac{\mathbf{g}_{\beta}^{(m)}}{\mathbf{I}_{\beta}+i \tau \mathbf{H}_{\beta \beta}^{(0)}} \mathbf{G}_{\beta \alpha}^{(n, n)}
$$

and

$$
\begin{aligned}
\mathbf{F}_{2 \alpha}^{(m, n)}= & -\tau^{2} \sum_{\beta=L, R} \sum_{j=0}^{m-1} \frac{\Lambda_{\beta}^{(m, j)}}{u_{\beta}^{(m)} u_{\beta}^{(j)}}\left(\mathbf{Q}_{\beta}^{(m-j)}\right. \\
& \left.+\mathbf{Q}_{\beta}^{(m-j-1)}\right)\left(\mathbf{G}_{C \alpha}^{(j+1, n)}+\mathbf{G}_{C \alpha}^{(j, n)}\right),
\end{aligned}
$$

where the propagators of the lead $\mathbf{g}_{\beta}^{(m)}$ and $\Lambda_{\beta}^{(m, j)}$ are defined as

$$
\mathbf{g}_{\beta}^{(m)}=\left[\frac{1-i \tau \mathbf{H}_{\beta \beta}^{(0)}}{1+i \tau \mathbf{H}_{\beta \beta}^{(0)}}\right]^{m}, \quad \Lambda_{\beta}^{(m, j)}=\prod_{l=j}^{m}\left(u_{\beta}^{(l)}\right)^{2},
$$

and

$$
\mathbf{Q}_{\beta}^{(m)}=\mathbf{H}_{C \beta} \frac{\mathbf{g}_{\beta}^{(m)}}{\mathbf{I}_{\beta}+i \tau \mathbf{H}_{\beta \beta}^{(0)}} \mathbf{H}_{\beta C} .
$$

By multiplying Eq. (25) by the auxiliary projection matrix $\overline{\boldsymbol{\Gamma}}_{\beta}$ from the right and setting $\alpha$ to be $C$, we immediately obtain the discretized version of Eq. (7) that conserves the charge density in the whole iteration process. Therefore, one can calculate $\mathbf{G}_{C C \beta}^{(m, n)}$ by iterating Eq. (25), which in turn gives the lesser Green's function and self-consistent Hartree potential. As mentioned in Sec. II, due to the huge computational cost of this exact NEGF-TDDFT approach, we would like to use the self-consistent Hartree potential to avoid the self-consistent loop.

We now show that this iteration procedure for the Green's function is very similar to that of the time-dependent wavefunction approach proposed in Ref. 21. According to Eq. (15), the wave function $\psi_{C}^{(m)}$ is given by

$$
\psi_{C}^{(m)}=i \sum_{\alpha=L, C, R} \mathbf{G}_{C \alpha}^{(m, 0)} \psi_{\alpha}^{(0)} .
$$

We wish to emphasize here that $\psi_{C}^{(m)}$ and $\psi_{\alpha}^{(0)}$ are the wave functions for a fixed energy. Combining Eqs. (25) and (30), it is straightforward to obtain an effective iterative equation of wave function in the central region,

$$
\left(\mathbf{I}_{C}+i \tau \mathbf{H}_{\mathrm{eff}}^{(m)}\right) \psi_{C}^{(m+1)}=\left(\mathbf{I}_{C}-i \tau \mathbf{H}_{\mathrm{eff}}^{(m)}\right) \psi_{C}^{(m)}+S^{(m)}+M^{(m)},
$$

where $S^{(m)}$ and $M^{(m)}$ are defined as

$$
S^{(m)}=-2 i \tau \sum_{\beta=L, R} \frac{\Lambda_{\beta}^{(m, 0)}}{u_{\beta}^{(m)}} \mathbf{H}_{C \beta} \frac{\mathbf{g}_{\beta}^{(m)}}{\mathbf{I}_{\beta}+i \tau \mathbf{H}_{\beta \beta}^{(0)}} \psi_{\beta}^{(0)}
$$

and

$$
\begin{aligned}
M^{(m)}= & -\tau^{2} \sum_{\beta=L, R} \sum_{j=0}^{m-1} \frac{\Lambda_{\beta}^{(m, j)}}{u_{\beta}^{(m)} u_{\beta}^{(j)}} \\
& \times\left(\mathbf{Q}_{\beta}^{(m-j)}+\mathbf{Q}_{\beta}^{(m-j-1)}\right)\left(\psi_{C}^{(j+1)}+\psi_{C}^{(j)}\right) .
\end{aligned}
$$

Equations (31)-(33) are first obtained in Ref. 21. In Eq. (31), two extra terms called the source term $S^{(m)}$ and memory term $M^{(m)}$ correspond to the first and second terms in Eq. (17). Here, the memory term accounts for electron hopping in and out of the central scattering region, while the source term is responsible for the injection of electron from the lead region $\alpha$ to the central scattering region.

Note that when we iterate Eq. (25) by setting $\alpha=C$, the "source term" $\mathbf{F}_{1 C}^{(m, n)}$ vanishes automatically since $\beta$ can only be $L, R$. Hence, Eq. (25) can be written as

$$
\left(\mathbf{I}_{C}+i \tau \mathbf{H}_{\mathrm{eff}}^{(m)}\right) \mathbf{G}_{C C}^{(m+1, n)}=\left(\mathbf{I}_{C}-i \tau \mathbf{H}_{\mathrm{eff}}^{(m)}\right) \mathbf{G}_{C C}^{(m, n)}+\mathbf{F}_{2 C}^{(m, n)}
$$

with the initial conditions for the "memory term" $\mathbf{F}_{2 C}^{(0,0)}=0$ and $\mathbf{F}_{2 C}^{(n, n)}$ for nonzero $n$ :

$$
\mathbf{F}_{2 C}^{(n, n)}=i \tau^{2} \sum_{\beta=L, R} \frac{\Lambda_{\beta}^{(n, n-1)}}{u_{\beta}^{(n)} u_{\beta}^{(n-1)}}\left(\mathbf{Q}_{\beta}^{(1)}+\mathbf{Q}_{\beta}^{(0)}\right),
$$

where we have used the fact that $\mathbf{G}_{C C}^{(n, n)}=-i$. Using $\mathbf{F}_{2 C}^{(n, n)}$ in Eq. (35), we can solve Eq. (34) for $\mathbf{G}_{C C}^{(n+1, n)}$, which is needed for $\mathbf{F}_{2 C}^{(n+1, n)}$ in Eq. (27). This procedure can be repeated until we obtain the whole solution. Since Eq. (27) involves the convolution with the Green's function of the previous time step, the computational complexity for calculating all the Green's functions $\mathbf{G}_{C C}^{r}(m \Delta t, n \Delta t)$ and hence the transient current is $O\left(N^{3}\right)$.

Now, we discuss how to iterate Eq. (31), which is more complicated than that of the Green's function discussed above. We first have to prepare the initial eigenstates of the equilibrium open device, i.e., in the absence of the time-dependent bias. In Ref. 21, the initial states of the central region are obtained from the diagonalization of the Green's function in the central region, which has to be rescaled by matching it with the known form of the wave function in the leads. However, one usually can not obtain an analytical form of wave function in the lead when dealing with atomic leads. Therefore, the method used in Ref. 21 is not very efficient in the first-principles calculation. Physically, it is natural to choose the scattering wave functions injected from leads as initial states, which are perfectly matched already. In the following, we will discuss in detail how to prepare these wave functions.

To begin with, we note that the eigenstates of the open device are composed of scattering wave states incidenting from left and right leads $\left\{\psi^{k_{L}^{n}}, \psi^{k_{R}^{n}}\right\}$ and a set of discrete bound states $\left\{\psi^{c^{n}}\right\}$. According to our previous discussion, we only consider 
scattering states

$$
\begin{aligned}
& \psi^{k_{L}^{n}} \equiv\left\{\begin{array}{l}
\psi_{L}^{k_{L}^{n}}=\varphi^{k_{L}^{n}}+\sum_{l} \varphi^{k_{L}^{l}} r^{k_{L}^{l} k_{L}^{n}}, \\
\psi_{C}^{k_{L}^{n}}, \\
\psi_{R}^{k_{L}^{n}}=\sum_{l} \varphi^{k_{R}^{l}} t^{k_{R}^{l} k_{L}^{n}},
\end{array}\right. \\
& \psi^{k_{R}^{n}} \equiv\left\{\begin{array}{l}
\psi_{L}^{k_{R}^{n}}=\sum_{l} \varphi^{k_{L}^{l}} t_{L}^{k_{L}^{l} k_{R}^{n}}, \\
\psi_{C}^{k_{R}^{n}}, \\
\psi_{R}^{k_{R}^{n}}=\varphi^{k_{R}^{n}}+\sum_{l} \varphi^{k_{R}^{l}} r_{R}^{k_{R}^{l} k_{R}^{n}}
\end{array}\right.
\end{aligned}
$$

where $\varphi^{k_{\alpha}^{n}}$ is the corresponding eigenchannel $n$ of the $\alpha$ lead, $n$ denotes the subband, and $k_{\alpha}$ is the Bloch wave vector of the $\alpha$ lead; $t^{k_{\beta}^{l} k_{\alpha}^{n}}$ and $r^{k_{\beta}^{l} k_{\alpha}^{n}}$ are transmission and reflection amplitudes from channel $n$ in the $\alpha$ lead to channel $l$ in the $\beta$ lead, respectively. In Eq. (36), the scattering state of the whole device is separated into three parts, and the scattering wave function in the central scattering region is labeled according to the eigenchannel injected from the $\alpha$ lead. In order to calculate the whole wave function, we first calculate the eigenchannel of the lead and that of the central region. After that, we match the boundary condition to find out the transmission and reflection amplitudes in Eq. (36).

We now calculate the eigenchannel wave function of each lead. Since the lead is semi-infinite periodic structure, the wave function in the $j$ th unit cell can be written as $\varphi^{k_{\alpha}^{n}}(j)=\phi^{k_{\alpha}^{n}} e^{i k_{\alpha}^{n} j}$ due to the Bloch theorem. The unit-cell wave function $\phi^{k_{\alpha}^{n}}$ can be obtained by solving

$$
\left[\mathbf{H}_{0 \alpha}+\mathbf{H}_{\alpha 1} e^{i k_{\alpha}^{n}}+\mathbf{H}_{1 \alpha} e^{-i k_{\alpha}^{n}}\right] \phi^{k_{\alpha}^{n}}=E \phi^{k_{\alpha}^{n}},
$$

which is equivalent to a quadratic eigenvalue problem ${ }^{39,40}$

$$
\begin{aligned}
& \left(\begin{array}{cc}
-\mathbf{H}_{\alpha 0}^{E} & -\mathbf{H}_{1 \alpha} \\
\mathbf{I}_{N_{\alpha}} & \mathbf{0}_{N_{\alpha}}
\end{array}\right)\left(\begin{array}{c}
\phi^{k_{\alpha}^{n}} \\
e^{-i k_{\alpha}^{n}} \phi^{k_{\alpha}^{n}}
\end{array}\right) \\
& =e^{i k_{\alpha}^{n}}\left(\begin{array}{cc}
\mathbf{H}_{\alpha 1} & \mathbf{0}_{N_{\alpha}} \\
\mathbf{0}_{N_{\alpha}} & \mathbf{I}_{N_{\alpha}}
\end{array}\right)\left(\begin{array}{c}
\phi^{k_{\alpha}^{n}} \\
e^{-i k_{\alpha}^{n}} \phi^{k_{\alpha}^{n}}
\end{array}\right),
\end{aligned}
$$

where we introduce $\mathbf{H}_{\alpha 0}^{E}=\mathbf{H}_{\alpha 0}-E \mathbf{I}_{N_{\alpha}}$ and $\mathbf{H}_{1 \alpha}=\mathbf{H}_{\alpha 1}^{\dagger}$ and $N_{\alpha}$ is the dimension of the unit cell. In general, there are $2 N_{\alpha}$ solutions for Eq. (28), which can be classified into $N_{\alpha}$ right-moving modes and $N_{\alpha}$ left-moving modes ${ }^{39}$ and can be labeled as $\phi^{k_{\alpha, \pm}^{n}}$, respectively. Total $N_{\alpha}$ modes include propagating modes and evanescent modes $\left(\left|e^{i k_{\alpha}^{n}}\right|<1\right.$ for the right-moving evanescent modes and $\left|e^{i k_{\alpha}^{n}}\right|>1$ for the left-moving evanescent modes). When $\left|e^{i k_{\alpha, \pm}^{n}}\right|$ is equal to 1 both for the left propagating modes and the right propagating modes, one has to use the sign of Bloch velocity to know whether it is left or right moving, which can be calculated from the following expression:

$$
v_{\alpha, \pm}^{n}=-2 \operatorname{Im}\left[e^{i k_{\alpha, \pm}^{n}}\left(\phi^{k_{\alpha, \pm}^{n}}\right)^{\dagger} \mathbf{H}_{\alpha 1} \phi^{k_{\alpha, \pm}^{n}}\right] .
$$

Note that eigenvectors are nonorthogonal in general; the dual vector $\widetilde{\phi}^{k_{\alpha}^{n}}$ is defined as

$$
\left(\widetilde{\phi}_{\alpha, \pm}^{k_{\alpha, \pm}^{n}}\right)^{\dagger} \phi^{k_{\alpha, \pm}^{m}}=\delta_{m n}, \quad\left(\phi^{k_{\alpha, \pm}^{n}}\right)^{\dagger} \widetilde{\phi}_{\alpha, \pm}^{k_{\alpha, \pm}}=\delta_{m n} .
$$

Physically, only right propagating channels injected from the left lead and left propagating channels from the right lead need to be considered as initial scattering states for the two-terminal device. Now, we are ready to calculate the wave function of the central scattering region. As shown in Refs. 41-43, it can be solved through following the linear equation

$$
\left(E_{k^{n}}-\mathbf{H}_{C C}-\sum_{\alpha} \boldsymbol{\Sigma}_{\alpha}^{r}\left(E_{k^{n}}\right)\right) \psi_{C}^{k_{\alpha}^{n}}=W^{k_{\alpha}^{n}},
$$

where $W^{k_{\alpha}^{n}}$ is the source term corresponding with propagating mode $n$ from the $\alpha$ lead. By introducing the auxiliary $j$ th power of Bloch matrices $\mathbf{B}_{\alpha, \pm}^{j}=\sum_{m=1}^{N} e^{i k_{\alpha, \pm}^{m} j} \phi^{k_{\alpha, \pm}^{m}} \widetilde{\phi}^{k_{\alpha, \pm}^{m}}$, the source can be expressed as ${ }^{44}$

$$
\begin{aligned}
& W^{k_{L}^{n}}=\left(\begin{array}{c}
\mathbf{H}_{1 L}\left[\mathbf{B}_{L,+}^{-1}-\mathbf{B}_{L,-}^{-1}\right] \phi^{k_{L,+}^{n}} \\
\cdot \\
\cdot \\
\dot{0}
\end{array}\right) \text {, } \\
& W_{R}^{k_{R}^{n}}=\left(\begin{array}{c}
\mathbf{0} \\
\cdot \\
\cdot \\
\cdot \\
\mathbf{H}_{R 1}\left[\mathbf{B}_{R,-}^{-1}-\mathbf{B}_{R,+}^{-1}\right] \phi^{k_{R,-}^{n}}
\end{array}\right) .
\end{aligned}
$$

Once Eq. (41) is solved, one can calculate the transmission and reflection amplitudes $t^{k_{\beta}^{l} k_{\alpha}^{n}}, r^{k_{\alpha}^{l} k_{\alpha}^{n}}$. For example, the transmission amplitude $t^{k_{R}^{l}} k_{L}^{n}$ is given by

$$
t^{k_{R}^{l} k_{L}^{n}}=\left(\widetilde{\phi}^{k_{R,+}^{l}}\right)^{\dagger} \psi_{C R}^{k_{L}^{n}},
$$

where $\psi_{C R}^{k_{L}^{n}}$ is a column vector denoting the part of the scattering wave function of the central region in contact with the right lead, while $\widetilde{\phi}^{k_{R,+}^{l}}$ is the column vector describing the wave function of the right lead. Similarly, the reflection amplitude $r^{k_{L}^{l}} k_{L}^{n}$ can be determined as

$$
r^{k_{L}^{l} k_{L}^{n}}=\left(\widetilde{\phi}^{k_{L,-}^{l}}\right)^{\dagger}\left(\psi_{C L}^{k_{L}^{n}}-\phi^{k_{L,+}^{l}}\right),
$$

where $\psi_{C L}^{k_{L}^{n}}$ denotes the wave function of the central region near the left lead. Note that the transmission and reflection amplitudes have to be normalized with velocities to form a unitary scattering matrix.

Once the initial wave function of the open device is known, we can calculate its time evolution and hence the time-dependent transient current according to Eq. (18). To do this, one has to know how to calculate $M^{(m)}$ and $S^{(m)}$. First, the calculation of memory term $M^{(m)}$ is straightforward when the quantity $\mathbf{Q}_{\alpha}^{(m)}$ in Eq. (29) is known. Due to the screening effect of the buffer layer in the central region, the scattering potential of the outermost buffer layers can be made very close to that of the periodic leads if the buffer layer is long enough. When this happens, the coupling matrix between the lead and the outermost buffer layer is $\mathbf{H}_{C \alpha}$. As a result, $\mathbf{Q}_{\alpha}^{(m)}$ should 
have the following structure:

$$
\mathbf{Q}_{L}^{(m)}=\left[\begin{array}{ccc}
\mathbf{q}_{L}^{(m)} & 0 & 0 \\
0 & 0 & 0 \\
0 & 0 & 0
\end{array}\right], \quad \mathbf{Q}_{R}^{(m)}=\left[\begin{array}{ccc}
0 & 0 & 0 \\
0 & 0 & 0 \\
0 & 0 & \mathbf{q}_{R}^{(m)}
\end{array}\right],
$$

where $\mathbf{q}_{\alpha}^{(m)}$ are $N_{\alpha} \times N_{\alpha}$ square matrices ${ }^{21}$

$$
\begin{aligned}
\mathbf{q}_{L}^{(m)} & =\mathbf{H}_{1 L}\left(\frac{\mathbf{g}_{L}^{(m)}}{\mathbf{I}_{L}+i \tau \mathbf{H}_{L L}^{(0)}}\right)_{1,1} \mathbf{H}_{L 1}, \\
\mathbf{q}_{R}^{(m)} & =\mathbf{H}_{R 1}\left(\frac{\mathbf{g}_{R}^{(m)}}{\mathbf{I}_{R}+i \tau \mathbf{H}_{R R}^{(0)}}\right)_{1,1} \mathbf{H}_{1 R} .
\end{aligned}
$$

They can be obtained through an iterative equation (for detail, please refer to Ref. 21). From Eq. (46), we see that $\mathbf{q}_{\alpha}^{(m)}$ only depends on the lead structure and is independent of initial wave function. Therefore, $\mathbf{q}_{\alpha}^{(m)}$ can be calculated once for all at the beginning of the numerical calculation.

Since $S^{(m)}$ involves multiplication of matrix of infinite size, it is important for us to simplify Eq. (32) first. As an example, we consider $S_{R}^{(m)}$, defined as

$$
S_{R}^{(m)}=\mathbf{H}_{C R} \frac{\mathbf{g}_{R}^{(m)}}{\mathbf{I}_{R}+i \tau \mathbf{H}_{R R}^{(0)}} \psi_{R}^{k_{L}^{n}},
$$

to illustrate how to calculate Eq. (32). Due to the relation ${ }^{21}$

$$
\begin{aligned}
& \frac{\mathbf{g}_{R}^{(m)}}{\mathbf{I}_{R}+i \tau \mathbf{H}_{R R}^{(0)}} \\
& \quad=\left.\frac{1}{m !}\left(-\frac{\partial}{\partial x}+\frac{\partial}{\partial y}\right)^{m}\left(\frac{1}{x \mathbf{I}_{R}+i y \tau \mathbf{H}_{R R}^{(0)}}\right)\right|_{x=y=1},
\end{aligned}
$$

we have

$$
S_{R}^{(m)}=\left.\frac{1}{m !}\left(-\frac{\partial}{\partial x}+\frac{\partial}{\partial y}\right)^{m} S_{R}(x, y)\right|_{x=y=1},
$$

with the generating function

$$
S_{R}(x, y)=\mathbf{H}_{C R} \frac{1}{x \mathbf{I}_{R}+i y \tau \mathbf{H}_{R R}^{(0)}} \psi_{R}^{k_{L}^{n}} .
$$

To calculate $S_{R}^{(m)}$, we can first calculate $S_{R}(x, y)$ :

$$
S_{R}(x, y)=\mathbf{H}_{C R}\left[\frac{1}{x}-\frac{1}{x} \frac{i y \tau \mathbf{H}_{R R}^{(0)}}{x \mathbf{I}_{R}+i y \tau \mathbf{H}_{R R}^{(0)}}\right] \psi_{R}^{k_{L}^{n}} .
$$

Note that $\psi_{R}^{k_{L}^{n}}$ is not the eigenfunction of Hamiltonian $\mathbf{H}_{R R}^{(0)}$. With the help of Eqs. (36) and (37), we can figure out how to calculate $\mathbf{H}_{R R}^{(0)} \psi_{R}^{k_{L}^{n}}$ :

$$
\begin{aligned}
& \mathbf{H}_{R R}^{(0)} \psi_{R}^{k_{L}^{n}}=\left[\begin{array}{cccc}
\mathbf{H}_{0 R} & \mathbf{H}_{R 1} & 0 & \ldots \\
\mathbf{H}_{1 R} & \mathbf{H}_{0 R} & \mathbf{H}_{R 1} & \ldots \\
0 & \mathbf{H}_{1 R} & \mathbf{H}_{0 R} & \ldots \\
\ldots & \ldots & \ldots & \ldots
\end{array}\right]\left[\begin{array}{c}
\sum_{l} t^{k_{R}^{l} k_{L}^{n}} \phi^{k_{R}^{l}} e^{i k_{R}^{l}} \\
\sum_{l} t_{R}^{k_{R}^{l} k_{L}^{n}} \phi^{k_{R}^{l}} e^{2 i k_{R}^{l}} \\
\sum_{l} t_{R}^{k_{R}^{l} k_{L}^{n}} \phi^{k_{R}^{l}} e^{3 i k_{R}^{l}} \\
\ldots
\end{array}\right] \\
& =E_{k_{L}^{n}} \psi_{R}^{k_{L}^{n}}-\left[\begin{array}{c}
\mathbf{H}_{1 R} \sum_{l} t^{k_{R}^{l} k_{L}^{n}} \phi^{k_{R}^{l}} \\
0 \\
0 \\
\cdots
\end{array}\right]
\end{aligned}
$$

where $E_{k_{L}^{n}}$ is the eigenenergy of incoming channel $n$ from the left lead. Therefore, Eq. (51) becomes

$$
\begin{aligned}
S_{R}(x, y)= & \mathbf{H}_{C R}\left[\frac{1}{x}-\frac{1}{x} \frac{i y \tau E_{k_{L}^{n}}}{x \mathbf{I}_{R}+i y \tau \mathbf{H}_{R R}^{(0)}}\right] \psi_{R}^{k_{L}^{n}} \\
& +i y \tau \frac{1}{x} \mathbf{H}_{C R} \frac{\mathbf{H}_{1 R}}{x \mathbf{I}_{R}+i y \tau \mathbf{H}_{R R}^{(0)}} \sum_{l} t^{k_{R}^{l} k_{L}^{n}} \phi^{k_{R}^{l}} .
\end{aligned}
$$

Due to the coupling matrix $\mathbf{H}_{C R}, S_{R}(x, y)$ and $S_{R}^{(m)}$ have the structure

$$
S_{R}(x, y)=\left[\begin{array}{c}
0 \\
0 \\
B(x, y)
\end{array}\right], \quad S_{R}^{(m)}=\left[\begin{array}{c}
0 \\
0 \\
B^{(m)}
\end{array}\right],
$$

where $B(x, y)$ and $B^{(m)}$ are $N_{R} \times 1$ vectors. By moving the second term on the right-hand side to the left side of Eq. (53), one can obtain

$$
B(x, y)=\frac{\mathbf{H}_{R 1} \sum_{l} t^{k_{R}^{l} k_{L}^{n}} e^{i k_{R}^{l}} \phi^{k_{R}^{l}}+i y \tau \mathbf{q}_{R}(x, y) \sum_{l} t^{k_{R}^{l} k_{L}^{n}} \phi^{k_{R}^{l}}}{x+i y \tau E_{k_{L}^{n}}},
$$

where $\mathbf{q}_{R}(x, y)$ is defined as

$$
\mathbf{q}_{R}(x, y)=\mathbf{H}_{R 1}\left(\frac{1}{x \mathbf{I}_{R}+i \tau y \mathbf{H}_{R R}^{(0)}}\right)_{1,1} \mathbf{H}_{1 R},
$$

and $\quad \mathbf{q}_{R}^{(m)}=\left.\frac{1}{m !}\left(-\frac{\partial}{\partial x}+\frac{\partial}{\partial y}\right)^{m} \mathbf{q}_{R}(x, y)\right|_{x=y=1}$. Consequently, $B^{(m)}$ can be calculated using Eq. (49):

$$
\begin{aligned}
B^{(m)}= & \mathbf{H}_{R 1} \frac{\left(1-i \tau E_{k_{L}^{n}}\right)^{m}}{\left(1+i \tau E_{k_{L}^{n}}\right)^{m+1}} \sum_{l} t^{k_{R}^{l} k_{L}^{n}} e^{i k_{R}^{l}} \phi^{k_{R}^{l}} \\
& +i \tau \sum_{j} \frac{\left(1-i \tau E_{k_{L}^{n}}\right)^{m-j}}{\left(1+i \tau E_{k_{L}^{n}}\right)^{m+1-j}}\left(\mathbf{q}_{R}^{(j)}\right. \\
& \left.+\mathbf{q}_{R}^{(j-1)}\right) \sum_{l} t^{k_{R}^{l} k_{L}^{n}} \phi^{k_{R}^{l}} .
\end{aligned}
$$

Another term $S_{L}^{(m)}=\left[\begin{array}{lll}\left(A^{(m)}\right)^{T} & 0 & 0\end{array}\right]^{T}$ can be derived in a similar way:

$$
\begin{aligned}
A^{(m)}= & \mathbf{H}_{1 L} \frac{\left(1-i \tau E_{k_{L}^{n}}\right)^{m}}{\left(1+i \tau E_{k_{L}^{n}}\right)^{m+1}}\left(\sum_{l} r^{k_{L}^{l} k_{L}^{n}} e^{-i k_{L}^{l}} \phi^{k_{L}^{l}}+e^{-i k_{L}^{n}} \phi^{k_{L}^{n}}\right) \\
& +i \tau \sum_{j} \frac{\left(1-i \tau E_{k_{L}^{n}}\right)^{m-j}}{\left(1+i \tau E_{k_{L}^{n}}\right)^{m+1-j}}\left(\mathbf{q}_{L}^{(j)}+\mathbf{q}_{L}^{(j-1)}\right) \\
& \times\left(\sum_{l} t^{k_{L}^{l} k_{L}^{n}} \phi^{k_{L}^{l}}+\phi^{k_{L}^{n}}\right) .
\end{aligned}
$$

Therefore, the source term for the wave function injected from the left lead can be calculated as $S^{(m)}=$ $-2 i \tau \sum_{\alpha=L, R} \frac{\Lambda_{\alpha}^{(m, 0)}}{u_{\alpha}^{(m)}} S_{\alpha}^{(m)}$.

It is worth mentioning that, in the above discussion, the orthogonal basis set is assumed to expand the Hamiltonian. So, one has to orthogonalize the basis set if the nonorthogonal basis such as the atomic orbital basis set (LCAO) is used. ${ }^{45}$

To summarize the key steps, we first need to calculate $\mathbf{q}_{\alpha}^{(m)}$, which is needed in $\mathbf{F}_{2 C}^{(m)}$ or $M^{(m)}$ and $S^{(m)}$ for the 
(a)

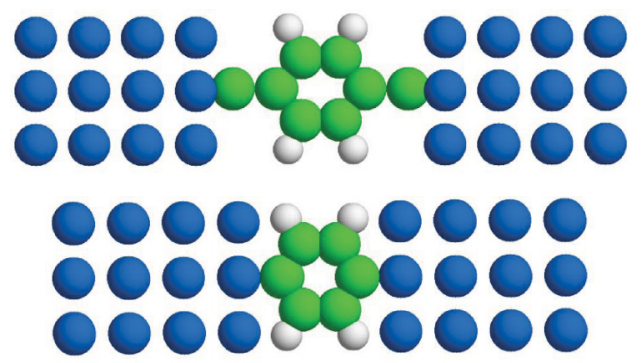

FIG. 2. (Color online) Schematic diagram of two molecular devices Al-1,4-dimethylbenzene-Al in panel (a) and Al-benzene-Al in panel (b). The device consists of 1,4-dimethylbenzene and benzene molecules coupled to perfect aluminium atomic electrodes along the (100) direction which extend to the reservoirs at $\pm \infty$.

iteration of Eqs. (34) or (31). For the NEGF-TDDFT approach, we simply iterate Eq. (34) for different $t^{\prime}<m \Delta t$. Once all the Green's functions are obtained, the lesser Green's function and the transient current are calculated from Eqs. (9) and (14), respectively. For the time-dependent wave-function approach, the following steps have to be done: (1) the initial scattering states coming from the left and right leads should be prepared; (2) the incoming states whose energy ranging from the band bottom to Fermi level should be included; (3) each scattering state is evolved using Eq. (31), and finally the time-dependent current can be calculated according to Eq. (18). Clearly, the NEGF-TDDFT approach is much simpler than the time-dependent wave function within the TDDFT approach.

\section{NUMERICAL RESULTS}

In this section, we implement our scheme and present numerical results of transient current for two molecular devices: 1,4-dimethylbenzene and benzene molecule coupled to two aluminum leads shown in Fig. 2. For these systems, there are 9 atoms in a unit cell with a finite cross section along the (100) direction in the semi-infinite aluminum lead. For the Al-1,4-dimethylbenzene-Al device, there are 66 atoms in the central scattering region and the distance between the $\mathrm{Al}$ atom and the nearest carbon atom is equal to 2.66 a.u. In the Al-benzene-Al device, there are 64 atoms in the central scattering region and the distance between the $\mathrm{Al}$ atom and the nearest carbon atom is equal to 3.0 a.u. The time step in the iteration is fixed as $\Delta t=0.01 \mathrm{fs}$.

Our numerical analysis is based on the state-of-the-art first-principles quantum transport package MATDCAL. ${ }^{46,47}$ Especially, a linear combination of atomic orbitals (LCAO) is employed to solve KS equations. The exchange-correlation is treated at the LDA level and the nonlocal norm-conserving pseudopotential ${ }^{48}$ is used to define the atomic core. The density matrix is constructed in orbital space and the effective potential is obtained in real space by solving the Poisson equation. The accuracy in the self-consistent iteration is numerically converged to $10^{-4} \mathrm{eV}$.

In the following, we will consider the upward steplike pulse that applies on both leads $\mathbf{V}_{L}(t)=-\mathbf{V}_{R}(t)=\theta(t) V$. In this case, $u_{\alpha}^{(m)}$ in Eq. (22) becomes a constant $\left.u_{\alpha}^{(m)}\right|_{m \geqslant 0}=u_{\alpha}=$ $\left(1+i \frac{\tau}{2} V\right) /\left(1-i \frac{\tau}{2} V\right)$. A recursive relation can be established

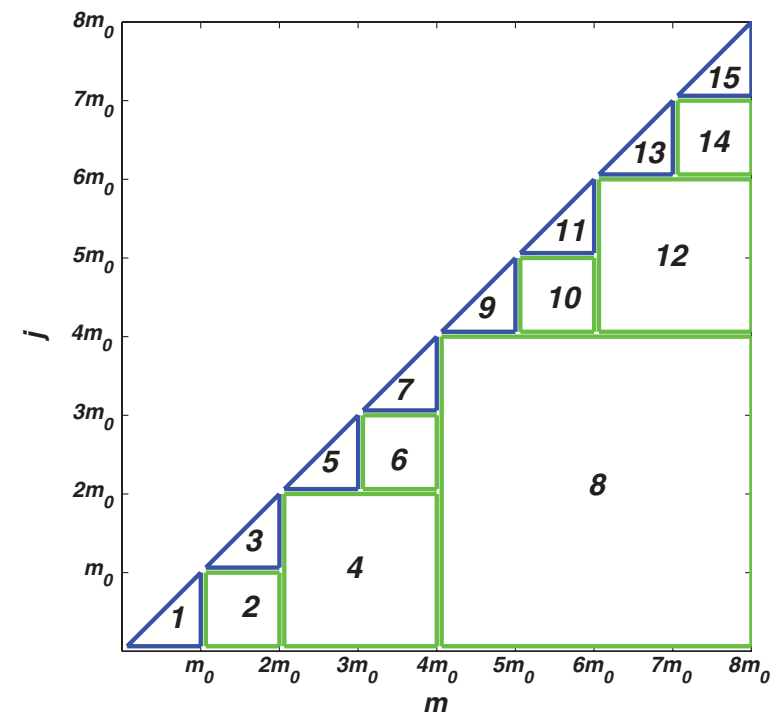

FIG. 3. (Color online) The schematic plot of computation procedures (from 1 to 15). Here, blue triangle represents direct calculation and green square represents FFT calculation.

for the source term $S^{(m)}$ and can be easily calculated, while the memory term $\mathbf{F}_{2 C}^{(m, n)}$ in Eq. (27) or $M^{(m)}$ in Eq. (33) becomes a discrete convolution summation

$$
\begin{aligned}
\mathbf{F}_{2 C}^{(m, n)}= & -\tau^{2} \sum_{\beta=L, R} \sum_{j=0}^{m-1}\left(u_{\beta}^{2}\right)^{m-j} \\
& \times\left(\mathbf{Q}_{\beta}^{(m-j)}+\mathbf{Q}_{\beta}^{(m-j-1)}\right)\left(\mathbf{G}_{C C}^{(j+1, n)}+\mathbf{G}_{C C}^{(j, n)}\right) .
\end{aligned}
$$

Similarly, $M^{(m)}$ in Eq. (33) is

$$
\begin{aligned}
M^{(m)}= & -\tau^{2} \sum_{\beta=L, R} \sum_{j=0}^{m-1}\left(u_{\beta}^{2}\right)^{m-j} \\
& \times\left(\mathbf{Q}_{\beta}^{(m-j)}+\mathbf{Q}_{\beta}^{(m-j-1)}\right)\left(\psi_{C}^{(j+1)}+\psi_{C}^{(j)}\right) .
\end{aligned}
$$

The method of fast Fourier transform (FFT) can be employed to reduce the computational cost. In particular, the computational complexity from $O\left(\mathrm{~m}^{3}\right)$ to $O\left[\mathrm{~m}^{2} \log _{2}^{2}(m)\right]$ can be achieved. ${ }^{49,50}$ In the following, we discuss how to use the FFT method in Eq. (60) to illustrate our approach. Note that the convolution in Eq. (60) is different from the traditional definition owing to the undetermined quantity $\psi_{C}^{(j)}$ in the kernel. Since the calculation $M^{(m)}$ in Eq. (60) has to be solved along with Eq. (31) for $m=1, \ldots, N$, we can choose $N=m_{0} 2^{n}$ where $m_{0}$ is a fixed number. Then, the whole calculation can be represented by the large triangle in Fig. 3 . The strategy is that some of the calculation in Eq. (60) will be done by direct summation over $j$ and the other will be done using FFT. The numerical scheme consists of several steps: (1) We start with a direct calculation of $M^{(m)}$ and $\psi_{C}^{(m)}$ using Eqs. (60) and (31) when $0<m \leqslant m_{0}$, which corresponds with the small triangle labeled as number 1 in Fig. 3. Usually, $m_{0}$ is chosen as certain power of 2 and can be optimized in the calculation. (2) To calculate $M^{(m)}$ for $m_{0}<m \leqslant 2 m_{0}$, we note that $\psi_{C}^{(j)}$ is known in the range $0<j \leqslant m_{0}$, the summation of $j$ in Eqs. (60) can be divided into two parts $0<m \leqslant m_{0}$ and $m_{0}<m \leqslant 2 m_{0}$. The first part corresponds to a square labeled 


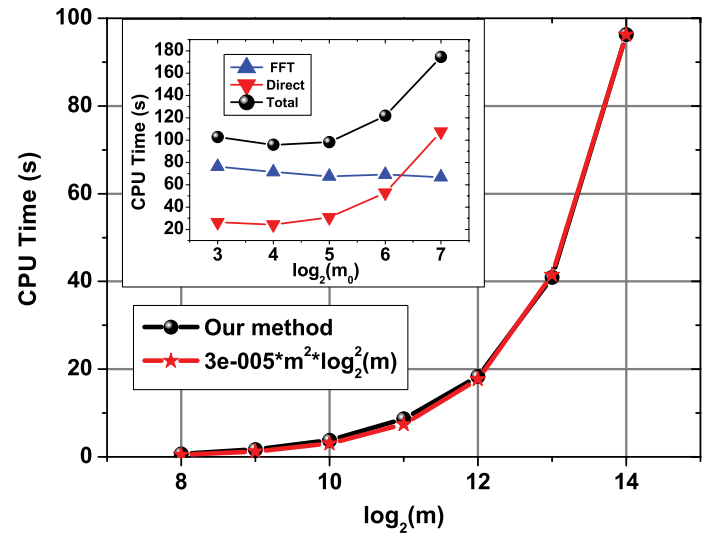

FIG. 4. (Color online) The scaling behavior of our numerical scheme with $m_{0}=16$. The inset shows CPU time versus $\log _{2}\left(m_{0}\right)$. Blue upper triangle represents CPU time of FFT calculation. Red lower triangle represents CPU time of direction calculation. Black sphere is the total CPU time.

as 2 in Fig. 3 and can be calculated using the FFT technique. The direct summation has to be done for the second part (the triangular labeled as 3 in Fig. 3). (3) The remaining steps are repeated FFT and direct calculations shown in Fig. 3 as squares and triangles, respectively.

To illustrate our numerical scheme, we take Al-1,4dimethylbenzene-Al device as an example. The optimized $m_{0}$ is determined through time evolution of one scattering state. Results are shown in the inset figure in Fig. 4. As we can see that the CPU time for FFT time decreases slowly as $m_{0}$ increases, while the direction calculation increases drastically with $m_{0}$. In practice, there is a minimum value for total CPU time consumed and $m_{0}=16$ in this case. After obtaining an optimized $m_{0}$, we then calculate the computational time of iterating a scattering wave with $m_{0}=16$ versus total number of time steps. The scaling behavior is exactly the $O\left[m^{2} \log _{2}^{2}(m)\right]$ shown in Fig. 4. According to our test, a speed-up factor of 300 when $m=16384$ has been gained by using the FFT technique.

As a check of our numerical implementation, we have calculated the transient current using two different methods. One is our present scheme named as the orbital-space method, while the other one is based on the time evolution of scattering wave function in $K$ space combined with DFT. The key idea of the $K$-space method is to transform the scattering state (extended in the whole space) of the open device from real space to $K$ space (localized in $K$ space). Then, the equation to solve the time-dependent $K$-space wave function becomes an integrodifferential equation. ${ }^{51}$ This scheme is also implemented into first-principles calculation by us. ${ }^{52}$ Here, we take the one-dimensional carbon atomic chain as a toy molecular device to test our implementation. In Fig. 5, the transient current calculated from two different methods agrees with with each other. In addition, in the long-time limit the transient current approaches the dc steady value obtained by using the Landauer-Büttiker formula.

Now, let us first study the Al-1,4-dimethylbenzene-Al device. The transient current $I(t)$ is shown in Fig. 6(a) ${ }^{53} \mathrm{We}$ see that the system is turned on at approximately $10 \mathrm{fs}$. As

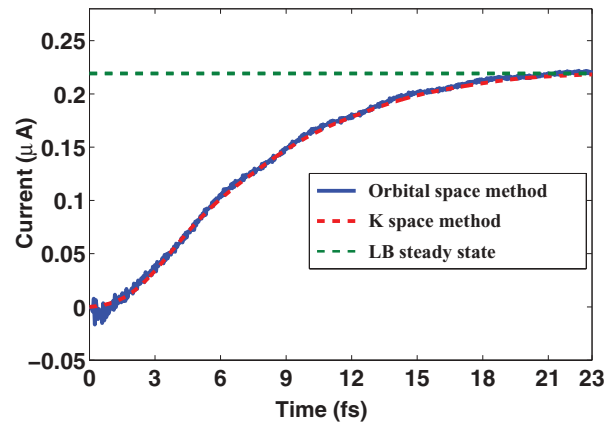

FIG. 5. (Color online) Time-dependent current $I(t)$ versus time with $V=0.0001$ a.u. for one-dimensional atomic carbon chain. Blue solid line and red dashed line are time-dependent current calculated by using the orbital-space method and the $K$-space method; green dashed line is the dc current at steady-state limit.

the device switches on, the transient current overshoots the dc limit and undergoes damped oscillation around it. We estimate that the relaxation time is roughly 150 fs. Physically, the damped oscillation is due to the presence of quasi-bound-state in the system that the incoming electron dwells for a long time. ${ }^{25,45}$ In Fig. 6(c), we show the largest contribution from one scattering state with $E=-0.03459$ a.u. is almost $40 \%$ of oscillatory transient current. To understand this behavior, we plot the transmission coefficient of equilibrium system shown in Fig. 6(d). Indeed, we see that at $E=-0.03459$ a.u., there is a sharp peak which is the signature of the quasi-bound-state. Finally, the transient current reaches the steady-state limit at around 300 fs.

In the second example, the transient current of the Albenzene-Al device is plotted in Fig. 7. For comparison, we calculate the transient current under two different bias voltages. We have several observations: (1) switch-on time is roughly $5 \mathrm{fs}$; (2) relaxation time is roughly $120 \mathrm{fs}$ for
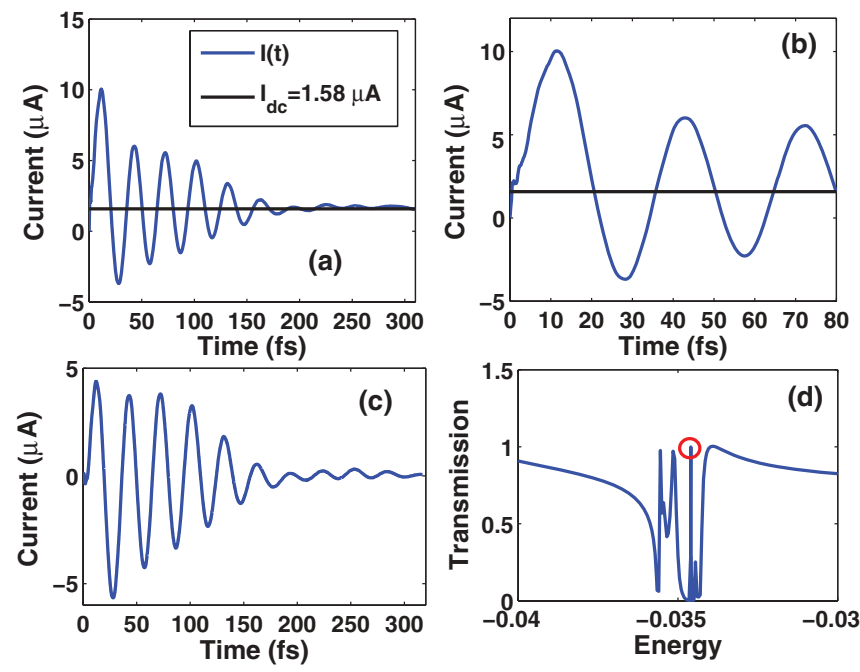

FIG. 6. (Color online) In panels (a) and (b), time-dependent current $I(t)$ versus time with $V=0.0025$ a.u. for Al-1,4dimethylbenzene-Al device are plotted [panel (b) is for a short-time scale]. Panel (c) shows the largest contribution to the oscillation from a particular scattering state with $E=-0.03459, k=1.81$. (d) Transmission coefficient when the system is in equilibrium state. 

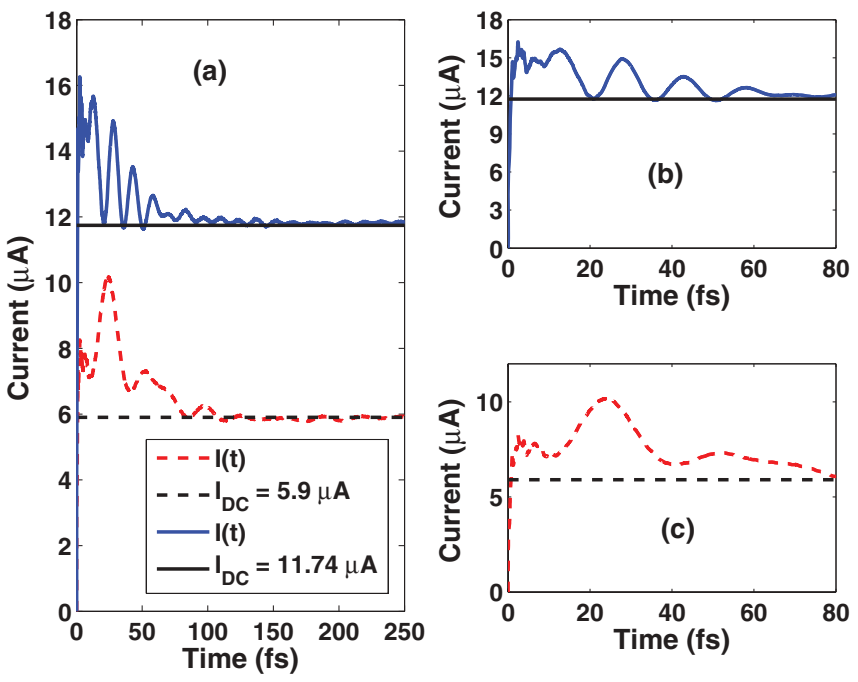

FIG. 7. (Color online) Time-dependent current $I(t)$ versus time with different bias voltages for Al-benzene-Al device. In panel (a), the dotted and solid lines correspond to $V=0.0025$ a.u. and $V=$ 0.005 a.u., respectively. Panels (b) and (C) show the early behavior of time-dependent current. Blue solid line and red dashed line are time-dependent current and black straight solid and dashed line are current at steady-state limit.

$V=0.0025$ a.u. and 180 fs for $V=0.005$ a.u.; (3) transient current is much larger than the dc limit at early time. As the bias voltage increases, the more oscillation occurs. In this device, the transient current overshoots the dc limit and then approaches the dc limit with less oscillation than that of the first device we studied.
As we see from the above results, the quasi-bound-state plays a dominant role in the transient current calculation of molecular devices. To resolve the quasi-bound-state spectral, one must go beyond the wideband-limit approximation so that essential physics of transient dynamics can be captured.

\section{SUMMARY}

In conclusion, we proposed a NEGF-TDDFT formalism to calculate the dynamical response of molecular devices due to the presence of external time-dependent bias on the lead. Detailed computational procedures for the first-principles transient current calculation were discussed. As an illustration, we have calculated the transient current for two molecular devices from first principles when the bias is a steplike pulse. By using the FFT technique, computational cost was drastically reduced from $N^{3}$ to $N^{2} \log _{2}^{2}(N)$. Comparing with the time-dependent wave function within TDDFT approach, our method is much easier to implement in the first-principles calculation in molecular devices.

\section{ACKNOWLEDGMENTS}

We gratefully acknowledge the support from Research Grant Council (HKU 705611P) and University Grant Council (Contract No. AoE/P-04/08) of the Government of HKSAR. Y. X. Xing is supported by the NSF-China under Grant No. 11174032 and Excellent young scholars Research Fund of Beijing Institute of Technology. This research is conducted using the HKU Computer Centre research computing facilities that are supported in part by the Hong Kong UGC Special Equipment Grant (SEG HKU09). *jianwang@hku.hk

${ }^{1}$ A. Aviram and M. Ratner, Chem. Phys. Lett. 29, 277 (1974).

${ }^{2}$ J. Chen, M. A. Reed, A. M. Rawlett, and J. M. Tour, Science 286, 1550 (1999).

${ }^{3}$ C. P. Collier et al., Science 285, 391 (1999).

${ }^{4}$ C. Joachim, J. K. Gimzewski, and A. Aviram, Nature (London) 408, 541 (2000).

${ }^{5}$ J. R. Heath and M. A. Ratner, Phys. Today 56(5), 43 (2003).

${ }^{6}$ J. R. Petta, S. K. Slater, and D. C. Ralph, Phys. Rev. Lett. 93, 136601 (2004).

${ }^{7}$ N. J. Tao, Nat. Nanotechnol. 1, 173 (2006).

${ }^{8}$ M. Di Ventra, S. T. Pantelides, and N. D. Lang, Phys. Rev. Lett. 84, 979 (2000).

${ }^{9}$ J. Taylor, H. Guo, and J. Wang, Phys. Rev. B 63, 245407 (2001); 63, 121104 (2001).

${ }^{10}$ M. Brandbyge, J. L. Mozos, P. Ordejon, J. Taylor, and K. Stokbro, Phys. Rev. B 65, 165401 (2002).

${ }^{11}$ Y. Xue, S. Datta, and M. A. Ratner, Chem. Phys. 281, 151 (2002).

${ }^{12}$ E. Louis, J. A. Verges, J. J. Palacios, A. J. Perez-Jimenez, and E. San Fabian, Phys. Rev. B 67, 155321 (2003).

${ }^{13}$ S.-H. Ke, H. U. Baranger, and W. Yang, Phys. Rev. B 70, 085410 (2004).

${ }^{14}$ C. C. Kaun, B. Larade, and H. Guo, Phys. Rev. B 67, 121411(R) (2003).
${ }^{15}$ C. C. Kaun and H. Guo, Nano Lett. 3, 1521 (2003).

${ }^{16}$ T. Frederiksen, M. Brandbyge, N. Lorente, and A.-P. Jauho, Phys. Rev. Lett. 93, 256601 (2004).

${ }^{17}$ T. Lee, W. Wang, J. F. Klemic, J. J. Zhang, J. Su, and M. A. Reed, J. Phys. Chem. B 108, 8742 (2004).

${ }^{18}$ Z. Y. Ning, W. Ji, and H. Guo, arXiv:0907.4674 (2010).

${ }^{19}$ E. Runge and E. K. U. Gross, Phys Rev Lett 52, 997 (1984).

${ }^{20}$ G. Stefanucci and C.-O. Almbladh, Phys. Rev. B 69, 195318 (2004).

${ }^{21}$ S. Kurth, G. Stefanucci, C.-O. Almbladh, A. Rubio, and E. K. U. Gross, Phys. Rev. B 72, 035308 (2005).

${ }^{22}$ G. Stefanucci, S. Kurth, E. K. U. Gross, and A. Rubio, Theor Comput. Chem. 17, 247 (2007).

${ }^{23}$ X. Zheng, F. Wang, C. Y. Yam, Y. Mo, and G. H. Chen, Phys. Rev. B 75, 195127 (2007).

${ }^{24}$ J. Maciejko, J. Wang, and H. Guo, Phys. Rev. B 74, 085324 (2006).

${ }^{25}$ B. Wang, Y. X. Xing, L. Zhang, and J. Wang, Phys. Rev. B 81, 121103(R) (2010).

${ }^{26}$ C. Caroli, R. Combescot, P. Nozeres, and D. Saint-James, J. Phys. C: Solid State Phys. 4, 916 (1971).

${ }^{27}$ C. Caroli, R. Combescot, D. Lederer, P. Nozeres, and D. SaintJames, J. Phys. C: Solid State Phys. 4, 2598 (1971).

${ }^{28}$ R. Combescot, J. Phys. C: Solid State Phys. 4, 2611 (1971).

${ }^{29}$ C. Caroli, R. Combescot, P. Nozieres, and D. Saint-James, J. Phys. C: Solid State Phys. 5, 21 (1972). 
${ }^{30}$ N. S. Wingreen, A.-P. Jauho, and Y. Meir, Phys. Rev. B 48, 8487 (1993); A.-P. Jauho, N. S. Wingreen, and Y. Meir, ibid. 50, 5528 (1994).

${ }^{31}$ M. Cini, Phys. Rev. B 22, 5887 (1980).

${ }^{32}$ Y. Zhu, J. Maciejko, T. Ji, H. Guo, and J. Wang, Phys. Rev. B 71, 075317 (2005).

${ }^{33}$ G. Stefanucci, S. Kurth, A. Rubio, and E. K. U. Gross, Phys. Rev. B 77, 075339 (2008).

${ }^{34} \mathrm{We}$ have used the adiabatic local density approximation on the exchange and correlation functional in TDDFT formalism.

${ }^{35}$ This fast algorithm actually gains speed by an additional factor of $M$, where $M$ is the dimension of the self-energy matrix of the lead and is about 30 in our calculation.

${ }^{36} \mathrm{H}$. Haug and A.-P. Jauho, Quantum Kinetics in Transport and Optics of Semiconductors (Springer, Berlin, 1998).

${ }^{37}$ C. Y. Yam, X. Zheng, G. H. Chen, Y. Wang, T. Frauenheim, and T. A. Niehaus, Phys. Rev. B 83, 245448 (2011).

${ }^{38}$ J. R. Hellums and W. R. Frensley, Phys. Rev. B 49, 2904 (1994).

${ }^{39}$ S. Sanvito, C. J. Lambert, J. H. Jefferson, and A. M. Bratkovsky, Phys. Rev. B 59, 11936 (1999).

${ }^{40}$ A. R. Rocha, V. M. García-Suárez, S. Bailey, C. Lambert, J. Ferrer, and S. Sanvito, Phys. Rev. B 73, 085414 (2006).
${ }^{41}$ P. A. Khomyakov, G. Brocks, V. Karpan, M. Zwierzycki, and P. J. Kelly, Phys. Rev. B 72, 035450 (2005).

${ }^{42}$ J. Wang and H. Guo, Phys. Rev. B 79, 045119 (2009).

${ }^{43}$ Hans Henrik B. Søensen, P. C. Hansen, D. E. Petersen, S. Skelboe, and K. Stokbro, Phys. Rev. B 79, 205322 (2009).

${ }^{44}$ G. Brocks, V. M. Karpan, P. J. Kelly, and P. A. Khomyakov et al., http://www.psik-k.org/newsletters/Nes_80/newsletter_80.pdf.

${ }^{45}$ Y. X. Xing, B. Wang, and J. Wang, Phys. Rev. B 82, 205112 (2010).

${ }^{46}$ D. Waldron, P. Haney, B. Larade, A. MacDonald, and H. Guo, Phys. Rev. Lett. 96, 166804 (2006).

${ }^{47}$ D. Waldron, V. Timoshevskii, Y. Hu, K. Xia, and H. Guo, Phys. Rev. Lett. 97, 226802 (2006).

${ }^{48}$ N. Troullier and J. L. Martins, Phys. Rev. B 43, 1993 (1991).

${ }^{49}$ E. Hairer, CH. Lubich, and M. Schlichte, SIAM J. Sci. Statist. Comput. 6, 532 (1985).

${ }^{50}$ I.-T. Chiang and W. C. Chew, IEEE Trans. Circuit Syst. II 52, 370 (2005).

${ }^{51}$ Z. Y. Zhou and S.-I. Chu, Europhys. Lett. 88, 17008 (2009).

${ }^{52}$ L. Zhang and J. Wang (unpublished).

${ }^{53}$ To ensure the current conservation, the time-dependent current $I(t)=\left[I_{R}(t)-I_{L}(t)\right] / 2$ is plotted (Ref. 45$)$. 